\title{
Pulmonary veno-occlusive disease in a unilateral hypertransradiant lung
}

\author{
M PAJEWSKI, R REIF, H MANOR, R STARINSKY, AND D KATZIR \\ From the Department of Radiology, Pathology, and Internal Medicine, Asaf Harofe Hospital, Zerifin, Israel.
}

Pulmonary veno-occlusive disease (VOD) is an extremely rare cause of pulmonary hypertension, leading ultimately to right heart failure and death. In the 30 or so cases published up to 1976 , the veno-occlusive process affected both lungs. 1

Calderon and Burdine ${ }^{2}$ reported a case affected by VOD with asymmetrical disturbance of perfusion caused by unilateral stenosis of major veins. The abnormalities of smaller veins and venules were, however, distributed in a similar fashion throughout both lungs.

This communication deals with a patient in whom VOD was confined to a unilateral hypertransradiant lung (Swyer-James MacLeod syndrome). The patient although suffering from recurrent bouts of unilateral lung infection was free of symptoms referrable to pulmonary hypertension. To the best of our knowledge no instance of a unilateral hypertransradiant lung being the site of pulmonary veno-occlusive disease has been reported.

\section{Case report}

This 21-year-old man was admitted to hospital with right chest pain, productive cough, and blood-stained sputum. He was a well-nourished man in good general condition. He smoked about 20 cigarettes daily for the last few years. There was no effort dyspnoea.

On physical examination some dullness over the right hemithorax was noted. Sedimentation rate, blood count, urinalysis, and ECG were normal. The Mantoux test was strongly positive. Lung function tests disclosed a very moderate mixed obstructive and restrictive function loss indicating small airway disease. The patient had been under the surveillance of a chest clinic since childhood because of recurrent pulmonary infections. On one occasion at the age of 16 years, because of active pulmonary tuberculosis in his family, he was treated with anti-tuberculous drugs for six months, even though the tuberculous nature of his affection was never proved. The chest radiograph showed widespread interstitial infiltration in the right middle and lower lung fields with obliteration of the costophrenic angle. On closer inspection one was impressed by the paucity of lung vascular markings, the smallness of the right hilum, and some loss of volume of the entire right lung (fig 1).

The patient received ampicillin and trimethoprimsulphamethoxasole for two weeks and the right lung

Address for reprint requests: Dr DM Pajewski, Department of Radiology, Pathology, and Internal Medicine, Asaf Harofe Hospital, Zerifin, Israel.

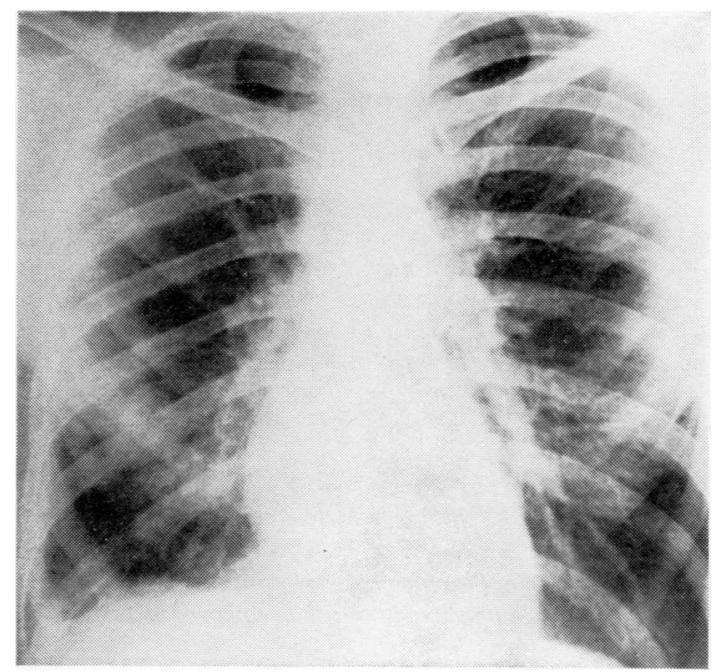

Fig 1 Chest radiograph on admission. Reduced size and deficient vascularity of the right lung. Streaky infiltration in the right lower and middle lung fields.

infiltration regressed. A perfusion scan disclosed no perfusion of the right lung. On bronchoscopy the major bronchi were patent, and on the right side some inflammatory changes in the mucosa were noted. Bronchography showed patchy parenchymatous filling especially in the right middle and lower lobes and obstructions in the fourth and fifth generations of bronchi. Pulmonary angiography displayed a markedly reduced diameter of the right pulmonary artery and an almost thread-like appearance of its branches. Venous return was absent on this side (figs 2 and 3 ).

At this stage, we traced a multitude of chest radiographs of the previous 10 years. There were recurrent bouts of infection with infiltrations always confined to the right lung. On one occasion at the age of 16 years a moderate right pleural effusion was present. In the intervals, the right lung was hypertransradiant and its vascular markings were deficient.

In order to ascertain the nature of the recurrent infiltration the patient had an open lung biopsy. Histology disclosed wide-spread changes in the pulmonary venules and veins. Intimal connective tissue proliferation with resulting luminal narrowing or obliteration was seen 


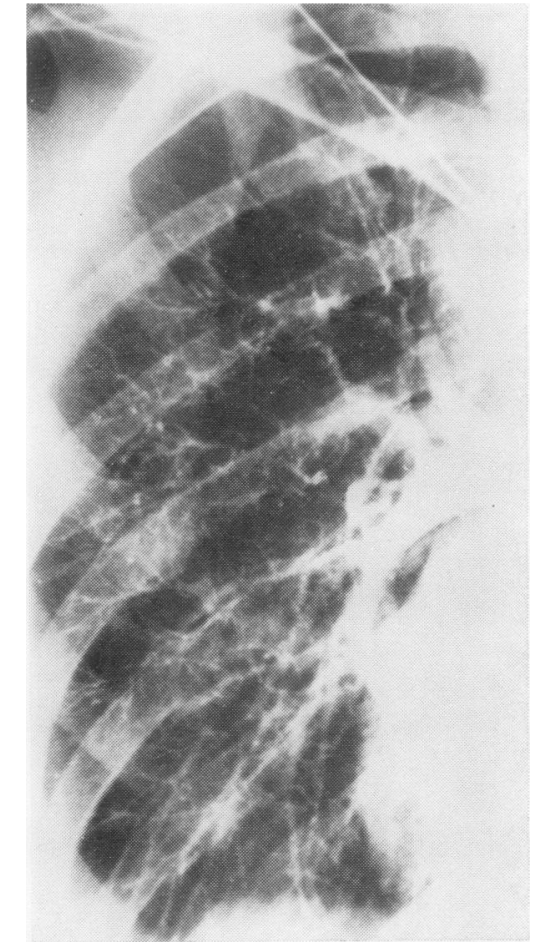

Fig 2 Direct injection into the right pulmonary artery shows threadlike appearance of the peripheral branches.

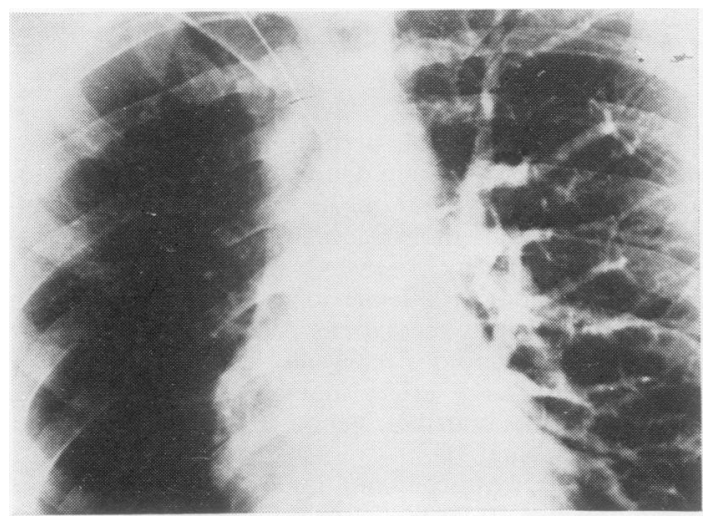

Fig 3 Venous phase. Venous return from the right lung is not seen.

throughout the entire biopsy (fig 4). The mural elastic layer was preserved in most of the venules and veins, but in some of them there was localised loss of elastic fibres. Thickened alveolar walls as a result of interstitial fibrosis were seen. There was no evidence of tuberculosis.

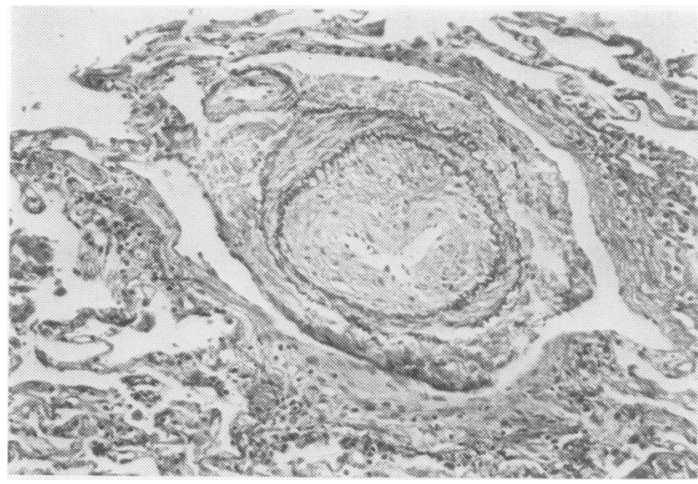

Fig 4. Pulmonary vein with nearly complete obliteration by intimal fibrous tissue The media is thickened, but the elastic fibres are preserved Elastic van Gieson, original magnification $\times 140$.

\section{Discussion}

The unilateral hypertransradiant lung is characterised radiologically by a small or normal sized organ which is poorly perfused. Its vessels are small in calibre. The major bronchi are characteristically patent, while signs indicating obstruction of smaller bronchi are always present with resulting insignificant changes of pulmonary density during all phases of respiration. Occasionally contralateral mediastinal shift on expiration is present. ${ }^{3-5}$

The chest radiographs of our patient over the past 10 years, perfusion study, bronchography, and pulmonary angiography leave little doubt about his harbouring a right hypertransradiant lung, which was the site of recurrent infections. The unusual feature in this case is the demonstration of a veno-occlusive process in a single lung with anatomically compromised structure. Venoocclusive disease restricts the venous bed of the lesser circulation with the resulting pulmonary venous hypertension. In our patient there were neither clinical nor radiological features of pulmonary venous hypertension, because of the unilateral localisation of the disease process.

The perfusion of the right lung already hampered by its basic pathology (hypertransradiance) was even more restricted by venous obstruction. The absent venous return from the right lung indicates that almost the entire output of the right ventricle was accommodated by the healthy lung. Although the contralateral lung was not biopsied, it seems to us that the lack of any clinical and radiographic evidence of pulmonary hypertension together with a normal ECG, normal left pulmonary angiogram and perfusion scan make the involvement of the latter most unlikely.

The aetiology of PVOD is unknown. A viral origin has been suggested, but clotting disorders and toxic factors may also play a role. ${ }^{2}$ The interstitial character of the recurrent infiltrations in this case favour, in our view, a viral aetiology. It is conceivable that the impaired circulation inherent in unilateral transradiant lung was a contributory factor leading to occlusion of small pulmonary veins. 
The unilateral transradiant lung is basically a benign condition. Therefore biopsies or resection specimens are rarely available. This might be the reason that PVOD has not been reported hitherto in this entity.

\section{References}

1 Wagenvoort CA. Pulmonary veno-occlusive disease. Chest 1976; 69:82-6.
2 Calderon M, Burdine JA. Pulmonary veno-occlusive disease $J$ Nucl Med 1974; 15:455-7.

3 Swyer PR, James GCW. A case of unilateral pulmonary emphysema Thorax $1953 ; 8$ : 133-6.

4 MacLeod WM. Abnormal transradiancy of one lung. Thorax 1954;9: 147-53.

5 Gotlieb S, Turner AF. Swyer-James (MacLeod) Syndrome. Variation in pulmonary bronchial arterial blood flow. Chest 1976; 69:62-6.

XI International Congress of Allergology and Clinical Immunology will be held under the Patronage of Her Majesty the Queen at the Barbican Centre, City of London from 17-22 October 1982. The scientific programme will include the following subjects: Mediators of Tissue Reactions; Paediatric Allergy-Influence of Ante and Neonatal Factors; Respiratory Allergy; Food Allergy; Animal/Insect Allergy; Clinical and Experimental Implications of IgE and IgG-STS Antibodies; Occupational Allergy; Vasculitis; Update on Antiallergic Drugs; Current Status of Immunotherapy; Diagnostic Procedures in Allergy. Further information may be obtained from Dr Robert J Davies/Professor Jack Pepys, Academic Unit of Respiratory Medicine, St Bartholomew's Hospital, West Smithfield, London EC1A 7BE or Conference Associates, 34 Stanford Road, London W8 5PZ. 